\title{
DESIGN OF ALGINATE BASED BLENDS FOR LIVING COMPOSITE FIBERS TO PROMOTE WOUND HEALING
}

\author{
Ceren OZEL ${ }^{1,6}$ (D), Tayfun SENGEL ${ }^{1,7}$ (iD, Aliakbar EBRAHIMI ${ }^{2,6}$ (D), Elif APAYDIN ${ }^{3,6}$ iD, \\ Hamed GHORBANPOOR ${ }^{4,6}$, Ayla EKER SARIBOYACI ${ }^{1,6}$ (D), Onur UYSAL ${ }^{1,6}$ (D), \\ Mete OZKURT ${ }^{5}$ (D), Huseyin AVCI ${ }^{2,6,8}$ (D) \\ ${ }^{1}$ Department of Stem Cell, Institute of Health Sciences, Eskisehir Osmangazi University, Eskisehir, Turkey \\ ${ }^{2}$ Department of Metallurgical and Materials Engineering,Eskişehir Osmangazi University Eskişehir, Turkey \\ ${ }^{3}$ Depertment of biochemistry, Institue of Health Sciences, Anadolu University, Eskişehir, Turkey \\ ${ }^{4}$ Department of Biomedical Engineering, Eskişehir Osmangazi University, Eskişehir, Turkey \\ ${ }^{5}$ Department of Physiology, Faculty of Medicine, Eskişehir Osmangazi University, Eskişehir, Turkey \\ ${ }^{6}$ Cellular Therapy and Stem Cell Production Application and Research Center (ESTEM), Eskişehir Osmangazi University, \\ Eskişehir, Turkey \\ ${ }^{7}$ Central Research Laboratory Research and Application Center (ARUM), Eskişehir Osmangazi University, \\ Eskişehir, Turkey \\ ${ }^{8}$ Translational Medicine Research and Clinical Center (TATUM), Eskişehir Osmangazi University, Eskişehir, Turkey
}

\begin{abstract}
The currently used approaches in the treatment of wounds and burns have been studied for many years to eliminate problems related with mechanical strength, elasticity, biocompatibility and cost. Nowadays, fabrication of composite fibers by a fiber as core and hydrogels as shell, which can be seeded by cells is rapidly increasing. In this study, it is aimed to produce a natural polymer-based dressing that can provide controlled antibiotic release to accelerate wound healing with low cost and high efficiency. The composites have been achieved by using surgical suture as a core and alginate in the shell part, which modified with starch and gelatin. Evaluating low-cost hydrogel material such as alginate, starch and gelatin in the shell layer of composite fibers by different concentrations were investigated in addition to study their swelling and drug release behaviors. The parameters for the model of an antibiotic release that can prevent common infections can be manipulated by using a biotextile-based approach to quantify the amount of antibiotics and its release to satisfy clinical requirements. Toluidine blue and Penicillin/Streptomycin were chosen as antibiotic models for drug release experiments. Moreover, human adipose tissue-derived mesenchymal stem cells (hAT-MSCs) were applied to evaluate cell viability experiments. Results demonstrated that alginate modified starch and gelatin can be used as low-cost and promisingmaterials for use in biomedical applications.
\end{abstract}

Keywords: Wound dressing, Composite fibers, Hydrogel, Core-shell, bio Textile

\section{INTRODUCTION}

The skin, known as the largest organ of the human body, is exposed to many external factors. Skin injury can be occurred by trauma, UV exposure, burns, and in some cases, wounds healing may take a long time and can lead to chronic wounds[1]. Since the recovery of the skin after injuries is a complex phenomenon that brings together many stages such as homeostasis, inflammation and reepithelialization [2]. In particular, various genetic disorders and chronic diseases such as type I diabetes or aging leads to chronic wounds, and that can make wound healing processes difficult [3]. The patients are generally suffering from leg and foot ulcers; their management requires the use of the wound dressings. Further, poor circulation also makes systemic administration of antibiotics ineffective and using high antibiotic doses leads to adverse effects in these patients. Only small local

*Corresponding Author:havci@ogu.edu.tr

Received: 20.08.2021 Published: 30.11.2021 
doses above the minimum inhibitory concentration are sufficient at the infected wound area. Finally, wound dressings that can provide recovery with systemic approaches in a short time are more advantageous than the expenses required for long-term wound care.

Developing fiber-based composite material for wound healingusing hydrogels is a promising platform in tissue engineering [4-7]. Hydrogels have a significant place among the biomaterials due to their high-water absorption capacity. In addition, hydrogels can reach deep wound tissues with minimal surgical intervention, and can also take the shape of the damaged area. Thus, they can create lower risk of scarring, inflammation, and infection in the tissues[8, 9]. For the last 30 years, alginate fibers play an important role in the wound care industry and known to have a positive impact. This is because it has specific properties that facilitate wound healing, high moisture absorption and ion exchange ability, excellent biocompatibility of material in fiber form and it is an easily obtainable natural material. Alginate fibers are one of the useful raw material, especially for highly absorbent wound dressings [10].

Sodium alginate is a member of compounds Generally Recognized as Safe (GRAS) compounds[11]. Sodium alginate is an inexpensive, biodegradable biomaterial with low antimicrobial properties, and extracted from seaweed. Its structure enables absorbing edema in the wound. Alginate based composite has great potential such providing microbial protection and stimulation of wound healing processes $[12,13]$. Alginate is known to form gel structures by covalent crosslinking with polycationic water-soluble polymers such as poly-L-lysine, chitosan, DEAE-dextran, amino-poly (oxyethylene) or proteins. Alginate shows a stable structure when cross-linked with $\mathrm{Ca}^{+2}$ as a multivalent cation. The viscosity of the alginate increases, as the $\mathrm{pH}$ decreases. For instance, the viscosity is maximized by forming hydrogen bonds at $\mathrm{pH}$ 3-3.5 due to the alginate skeleton becomes protonated. This is generally undesirable situation because during the mixing and injection stages of cell encapsulation,tensile stress from high viscosity can occur, which can damage cells or protein structures [13-15]. Alginate dressing maintains a physiologically moist micro-environment, minimizes bacterial infection in the wound area and treats the wound. These gels also ensure that the newly formed granulation tissue is not disrupted and the skin surrounding the wound is not damaged. Moreover, they act as an effective hemostatic agent in bleeding wounds. Alginates can absorb liquid up to 15-20 times their weight and make them an ideal dressing material for wounds with high exudate $[10,11]$. In this way, they can stay on the wound for several days, thus minimizing the need for dressing changes. In comparative studies with hydrocolloid bandages, alginate dressings have been shown to be better in terms of fluid control, gel blocking, pain control and healing properties [16].

Alginate gels are promising for cell transplantation in tissue engineering. In this approach, hydrogels deliver cells to the desired site, provide space for new tissue formation, and control the structure and function of the treated tissue. As another property, alginate can release drug molecules from small chemical drugs up to macromolecular proteins, which is possible depending on the type and method of the cross-linking. In addition to the superior properties of the alginate, various additives can be added to the alginate such as starch and gelatin in order to control the desired drug release, strength and degradation processes [17].

Starch is a material composed from amylose and amylopectin. When the starch is heated in the presence of water, it undergoes a process known as gelatinisation, in which the granules swell, leach amylopectin and lose birefringence [18]. Studies have shown that it is a suitable material for different applications due to its biodegradability, easy availability and economic advantages. Starch stands out as risk-free and low-cost alternative material, which plays role in increasing cellular activity, differentiation and tissue remodeling among the materials used in scaffold fabrication [4, 19, 20]. Also, starch offers excellent potential for crosslinking due to the presence of abundant hydroxyl $(-\mathrm{OH})$ groups and has proven to be a good precursor material for the preparation of hydrogels [21, 22]. 
However, its highly hydrophilic nature and poor mechanical properties have hindered its usage in tissue engineering compared to some expensive, commercial polymers [23]. In addition, gelatin is a biocompatible protein, which is used to prepare composite films for biomedical applications due to its low cost, excellent functional and filmogenic properties. Gelatin is obtained by treating collagencontaining tissues with acid and/or alkali and then heat-treating in the presence of water to irreversibly break the collagen fibrillary structure. Gelatin is a material with a distinctive odor, colorless or slightly yellow, suitable for use found in the form of strips, powders or granules. Gelatin derivatives are one of the most efficient materials used in wound healing, which is a natural component of human skin [24, 25]. In addition, gelatinderivatives have advantageous in absorbing excess exudates due to its excellent ability to absorb water 5-10 times more than its own weight [26]. So, they are quite suitable for use as a composite material due to their distinct properties of being natural hydrogel.

A composite material is a combination of two or more specialized materials to do a specific function with different physical and chemical properties, without dissolving or blending them into each other [27]. Investigations for development of cell-laden composite fiber have gained momentum in recent years. The reason behind this is that these composite fibers possess compositional and structural multifunctionality. Cell-laden composite fibers have core-shell structure in which the core part is biologically relevant in terms of mechanical properties and/or consisting of biodegradable polymer fibrils, and the shell part comprised of living cells and hydrogels which contains other bioactive agents such as growth factors or antibiotics [28-30]. This core/shell architecture provides a mechanically weak scaffold especially considering the shell part, while it also prevents the risk of fracture of fibers by benefitting from the strength of the fibers [30]. Loading of cells on the shell part is particularly important for the replacement of the lost tissues and for their regeneration with secreted bioactive molecules $[28,30]$. Furthermore, the core/shell structure allows the formation of hydrogel which brings many advantages: Hydrogels are the hydrophilic, biodegradable and biocompatible, can bear bioactive components and encapsulate the cells, can be modified easily and be used as delivery vehicle.

In the design of this study, various combinations of alginate, gelatin and starch were used to determine the optimum structural combination for the most appropriate drug release profile. The swelling rates and drug release profiles of these hydrogels and viability of hAT--MSCs in hydrogels coated to surgical sutures as core/shell compositeswere studied and structure of core/shell composites were imagined by SEM techniques.

\section{MATERIAL AND METHODS}

\subsection{Preparation of Alginate and Mixtures}

Alginate (low viscosity sodium alginate derived from brown algae, Sigma), starch (Cottonal KS) and gelatin (Gelatin from bovine skin, Sigma) used in our study were prepared using double distilled water at different concentrations $(\mathrm{w} / \mathrm{w})$. Alginate was prepared as different variety concentrations between 1$5 \%(\mathrm{w} / \mathrm{v})$. Alginate blends were prepared with gelatin and starch by adding to the pre-polymer alginate solutions at a ratio between 1-3\%. While preparing alginate and gelatin, the preparation was made by mixing at $50^{\circ} \mathrm{C}$ for 2 hours at a speed of $750-1000 \mathrm{rpm}$. The obtained mixtures were cross-linked using $0.2 \mathrm{M} \mathrm{CaCl}_{2}$ prepared in double distilled water.

\subsection{Performing Swelling Experiments}

The prepared mixtures were first polymerized to produce hydrogels for swelling experiments. After the polymerization process, the excess water on the obtained hydrogels was removed by means of 
blotting paper, and the hydrogels were weighted. The liquid loss of the hydrogels was achieved by keeping them in an incubator at $37^{\circ} \mathrm{C}$ for 24 hours. After 24 hours, dry weights $\left(\mathrm{M}_{\text {dry }}\right)$ of the hydrogelswere recorded. Then, each hydrogel was taken into $100 \mathrm{~mL}$ of double distilled water and allowed to swell at $37^{\circ} \mathrm{C}$ for 24 hours. At the end of 24 hours, the excess water of the hydrogels wereremoved with blotting paper. The wet weight of hydrogels $\left(\mathrm{M}_{\mathrm{wet}}\right)$ were obtained and recorded.Swelling calculations were made by using the obtained weights in the following equation:

$$
\text { Swelling Ratio }=\frac{M_{w e t}-M_{d r y}}{M_{d r y}} \times 100
$$

\subsection{Scanning Electron Microscopic Imaging}

The prepared alginate hydrogel was pulled around the 3-0 surgical suture to form core/shell layers(core: surgical suture, shell: hydrogel). A commercial absorbable Poly(glycolide/L-Lactide) surgical suture (MITSUTM POLYGLACTIN 910) which is composed of a copolymer made $90 \%$ Glycolide and 10\% L-Lactide, with a size of 3-0, was used as a core part of core/shell form. The surgical suture is coated with hydrogel by passing through in-house with a custom-built needles/tips having a flow rate of $2 \mathrm{ml} / \mathrm{min}$. The composite was then kept in $0.2 \mathrm{M} \mathrm{CaCl}_{2}$ solution for 15 minutes for crosslinking process and after that washed 3 times with PBS. After this stage, the sample was left to dry in a graduated ethanol series $(30-50-70-90-100)$ under hood. After the sample was fixed on the stub using carbon tape, they were coated with $4 \mathrm{~nm}$ gold/palladium (Leica EM CPD300). Then the sample wasanalyzed by scanning electron microscope device (SEM, Hitachi Regulus 8230) at $1 \mathrm{kV}$ voltage.

\subsection{Toluidine Blue and Antibiotic Release from Alginate Hydrogels}

The release of Toluidine blue (sigma) and Penicillin/streptomycin (Gibco) from the alginate and alginate doped gelation or starch hydrogels were investigated. For this aim, different ratio of aqueous mixtures of alginate, and different mixtures of alginate with gelatin or starch were prepared. 1 gr Toluidine blue was added to $10 \mathrm{ml}$ of each mixtureor $1 \mathrm{ml}$ of Penicillin/streptomycin $(10,000 \mathrm{units} / \mathrm{mL}$ penicillin $/ 10,000 \mu \mathrm{g} / \mathrm{mL}$ streptomycin) was added to $9 \mathrm{ml}$ of each mixture and mixed at $500 \mathrm{rpm}$ in a dark environment for 2 hours at $40^{\circ} \mathrm{C}$. After mixing process, the samples were cross-linked in $0.2 \mathrm{M}$ $\mathrm{CaCl}_{2}$ solution for 15 minutes and then allowed to release for 24 hours at room temperature. Then, the samples were analyzed by UV-Vis spectrophotometer (A\&ELAB).

\subsection{Demonstration of Cellular Encapsulation on the Composite Fibers}

A red PKH26 (Sigma) fluorescent dye were used to stain hAT-MSCs according to the manufacturer's protocol before encapsulation with hydrogels. Mesenchymal stem cells $\left(500 \times 10^{5}\right.$ cells $\left./ \mathrm{ml}\right)$ stained with PKH26 (Sigma) fluorescent dye were added to alginate pre-polymer hydrogel mixture and homogenized, which was followed by coating of the surgical sutures with the same method described "living core-shell fiber preparation" Afterwards, the formation of cell-laden hydrogel shell layer was obtained.

\subsection{Cell Viability/Cytotoxicity Test}

In order to examine cell viability within composite living fibers, isolated and characterized human adipose tissue-derived mesenchymal stem cells (hAT-MSCs) were used from our previous studieswhich hadbeen isolated and cryopreservedfrom informed/consented lipoaspirates of healthy donors undergoing elective liposuction, under Eskişehir Osmangazi University Clinical Research Ethics Committee permission (no: 80558721/231, 09/06/2017-12). hAT-MSCs were cultured and 
characterized as in previously described protocols depending in vitro differentiation experiments and immunophenotyping by flow cytometry[30-32]. hAT- MSCs were thawed and cultured in 75 $\mathrm{cm}^{2}$ dishes, at $37{ }^{\circ} \mathrm{C}$ and $5 \% \mathrm{CO}_{2}$ conditions for their expansion.For this aim, hAT-MSCs werecollected after centrifugation and plated in completeDMEM-F12 MSC culture medium containing $10 \%$ FBS and $1 \%$ penicillin-streptomycin and $1 \%(\mathrm{~mL} / \mathrm{mL})$ stable L. -Glutamine (Glutamax, Sigma), 15mM HEPES and Dulbecco's Modified Eagle Medium (DMEM-F12, Sigma), and the cells incubated at $37^{\circ} \mathrm{C}$ in $5 \% \mathrm{CO}_{2}$ and $\% 99$ relatively humidified incubator.

Fluorescent live/dead staining (Live/Dead (AAT Bioquest ${ }^{\circledR}$ ) was used to determine the viability of hAT-MSCs within composite living fibers after 24 hours in hAT-MSC culture mediumat $37^{\circ} \mathrm{C}$ in $5 \%$ CO2. For this purpose, after washing the samples 3 times with PBS, they were incubated in the dark for 1hour with $1 \mu \mathrm{M}$ Calcein-AM and $2 \mu \mathrm{M}$ Propidium Iodide. Live (green) and dead (red) cells were visualized under a fluorescent microscope. Calcein-AM green was stimulated in the FITC channel, and Propidium iodide red in the Texas Red channel.

\section{RESULTS AND DISCUSSION}

In our study, different concentrations of alginate mixtures (alginate alone or alginate + starch or gelatin) were prepared and after crosslinking process (hydrogel formation), swelling, release and cellular survival results of them were investigated. In this study, it was aimed to control the swelling profile of hydrogels with different density of alginate and also by adding starch or gelatin. Swellingratesobtained for $1 \%, 3 \%$ and $5 \%$ of alginatewere respectively $2634.78 ; 2488.89$ and 5460 (Table 1).

Alginate is composed of mannuronic and guluronic acid units [32]. Guluronic acid densed alginate has a high viscosity and will swell less because it will bind to more calcium units [33]. When alginate gels are placed in saline, $\mathrm{Na}^{+}$ions in the swelling medium enter inside hydrogel and undergo ion exchange with $\mathrm{Ca}^{++}$ions bound to the -COO- groups of 1,4-linked $\beta$-D-mannuronic (M) blocks. This, along with increased swelling, causes the $\mathrm{M}$ chains to relax. The external $\mathrm{Na}^{+}$ions then enter the 'egg-box' spaces and replace the existing $\mathrm{Ca}^{++}$ions. This also results in facial swelling. The fully hydrated structure begins to lose its structural integrity due to disruption of the 'egg box' cavities and the alginate chains begin to break down and dissolve [34]. The observed increases in swelling rate for 5\% alginate hydrogel may be due to the anionic nature of alginate, increase in density of alginate will cause increased repulsion between the molecular chains, resulting in an increase in swelling rate [35].

Table 1. Swelling data of surgical sutures covered with alginate and various additives.

\begin{tabular}{|c|c|c|c|}
\hline Mixture & Dry Weight (g) & $\begin{array}{c}\text { Swelling Weight } \\
(\mathbf{g})\end{array}$ & Swelling Rate \\
\hline 1\% Alginate & 0.23 & 6.29 & 2634.78 \\
\hline 1\%Alginate + 1\% Gelatin & 0.085 & 0.132 & 55.29 \\
\hline 1\% Alginate +1\%Starch & 0.33 & 1.09 & 230.30 \\
\hline 2\% Alginate +2\% Gelatin & 0.029 & 0.103 & 255.17 \\
\hline 2\% Alginate + 2\% Starch & 0.53 & 2.75 & 418.87 \\
\hline 3\% Alginate & 0.27 & 6.99 & 2488.89 \\
\hline 3\% Alginate + 2\% Gelatin & 0.091 & 0.871 & 857.14 \\
\hline 3\% Alginate + 2\% Starch & 0.067 & 1.28 & 1810.45 \\
\hline 5\% Alginate & 0.125 & 6.95 & 5460 \\
\hline 5\% Alginate + 1\% Gelatin & 0.156 & 8.15 & 5124.36 \\
\hline
\end{tabular}


According to data obtained for alginate-gelatin hydrogels, the addition of gelatin to the alginate provides a significant control of theswelling rates depending on the percentage of gelatin in the mixture. When $1 \%$ gelatin was added to $1 \%$ alginate solution, the swelling ratio of the obtained hydrogel was 55.29 . This rate is approximately $2 \%$ of the swelling rate of $1 \%$ alginate. Also, for $3 \%$ alginate, $\% 2$ gelatin mixture hydrogel swelling rate is $34 \%$ of the swelling rate of $3 \%$ alginate.As another group in this study, the swelling ratio of the hydrogel obtained by adding $1 \%$ gelatin to $5 \%$ alginate solution was 5124.36. This swelling rate is $94 \%$ of the swelling rate of $5 \%$ Alginate hydrogel.Yao et al. (2012) that obtained microspheres from mixtures of alginate and gelatin at different concentrations in their study, revealed that alginate was the main gelation factor for the obtained spheres and suggested that less stable and lower strength spheres were formed [36]. Here, 2 profiles were detected for alginate-gelatin hydrogels. The first one is the increase in swelling rate with increase in total density of polymers (total polymers density: 2\%, 5\%, 6\%, swelling rate: $55.29<$ $857.14<5124.36)$. This result is the same as the result obtained for alginate hydrogels. The second one is decreasing in swelling rate by adding gelatin to alginate.In a study conducted by Pulat in 2018, it was shown that the swelling rate decreases as the gelatin concentration increases[37].

When the ratio of alginate and gelatin is the same (1\% alginate $+1 \%$ gelatin), sharply decrease in swelling rate in compare with alginate (1\%) was detected (from 2634.78 to 55.29), and by increasing of the ratio of alginate to gelatin in hydrogelcomposition, the swelling ratio in compare with alginate hydrogels was increased $(2 \%<34 \%<94 \%)$. These results show that when the ratio of alginate is dominant to gelatin $(5 \%: 1 \%)$ the swelling rate of the hydrogel on a large scale controlled by alginate ( $94 \%$ of the swelling rate of $5 \%$ alginate).

When $1 \%$ starch is added to the $1 \%$ alginate solution, the swelling rate of the prepared hydrogel was found to be approximately $9 \%$ of the $1 \%$ alginate hydrogel. The same as gelatin doped alginate hydrogels, adding of starch with the same ratio of alginate to hydrogel composition (1\% alginate: $1 \%$ starch) cause to a sharp decrease in swelling ratio in comparewith $1 \%$ alginate hydrogel. By increasing the ratio of the alginate in hydrogels composition, increase in swelling ratio of hydrogels were detected (alginate/gelatin ratio: 1, 1.5, swelling rate: $230.30<1810.45$ ). These results show that adding starch to alginate has the same profile detected for adding of gelatin. Increasing in the ratio of starch in hydrogel composition cause todecrease in swelling rate.

Swelling behaviors of a hydrogel depend on composition, monomer ratio, ionic charge content, and polymerization route, type and density of crosslinker[38].By increasingthe degree of crosslinking, alginate will significantly reduce its swelling in the presence of solvent, resulting in reduced permeability of different solutes. As a result, the release of encapsulated drugs in alginate matrices will be delayed by increase of the degree of crosslinking and as a result itis suitable for use in controlled drug release applications[39]. Also, according to swelling results for hydrogels prepared by mixing different ratios of alginate with starch or gelatin, it has been shown that it is possible to control the swelling properties of the alginate based hydrogels (Figure 1). The data obtained from the experiments showed that gelatin caused a greater decrease in swelling data compared to starch. 


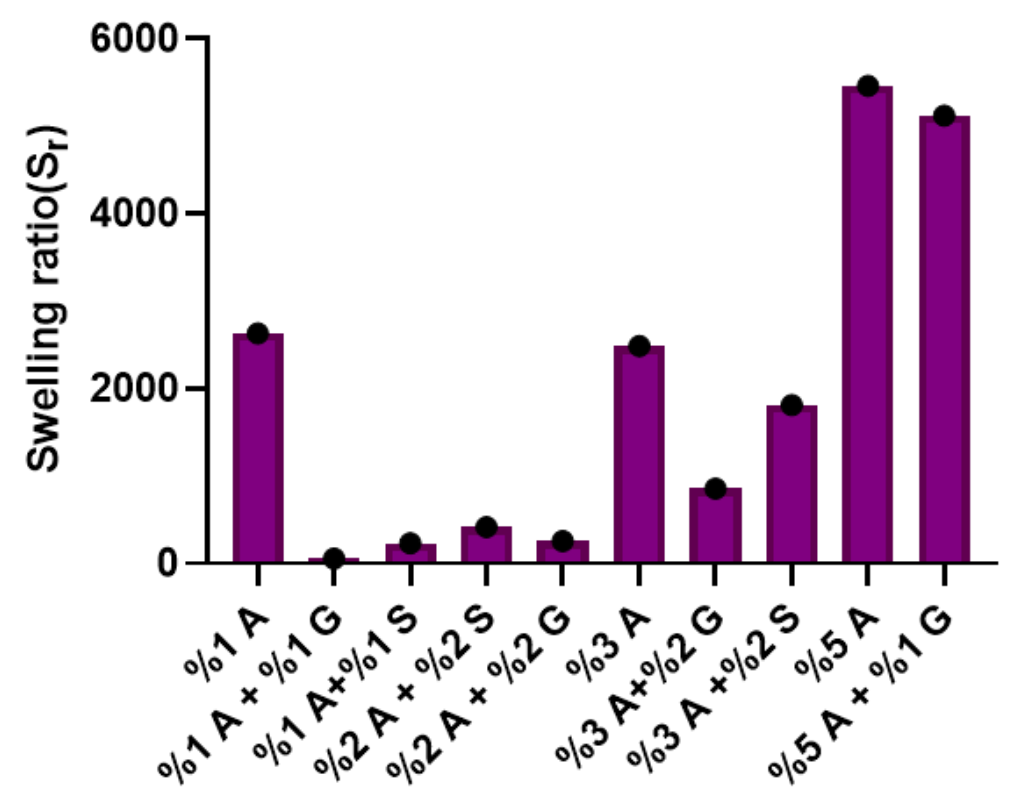

Figure 1. Swelling ratio of surgical sutures coated with alginate hydrogels or hydrogels composed of different mixtures of alginate with starch or gelatin. A: Alginate, G: Gelatin, S: Starch.

Although starch is thought to increase hydrophilic propertyof the hydrogel due to its hydrophilic nature, the complex macromolecular chain sequence obtained as a result of mixing can delay the penetration of water molecules into the hydrogel and therefore reduce the swelling rate. In hydrogels consisting of the mixture of starch and alginate, a high degree of cross-linking between molecules result in reduced swelling rates [35].

Structural analyzes of surgical sutures coated with alginate were made by SEM imaging. Analysis with 3-0 suture samples coated with antibiotic-loaded alginate hydrogel show theformation of hydrogel layer around the sutures. Analyzes performed using Image J (version 1.53k), showed that the diameter of the surgical suturesbefore then coating was $340 \mu \mathrm{m}$ and the thickness after hydrogel coating was $390 \mu \mathrm{m}$ (Figure 2). The thickness of the coating layer was considered to be $25 \mu \mathrm{m}$.

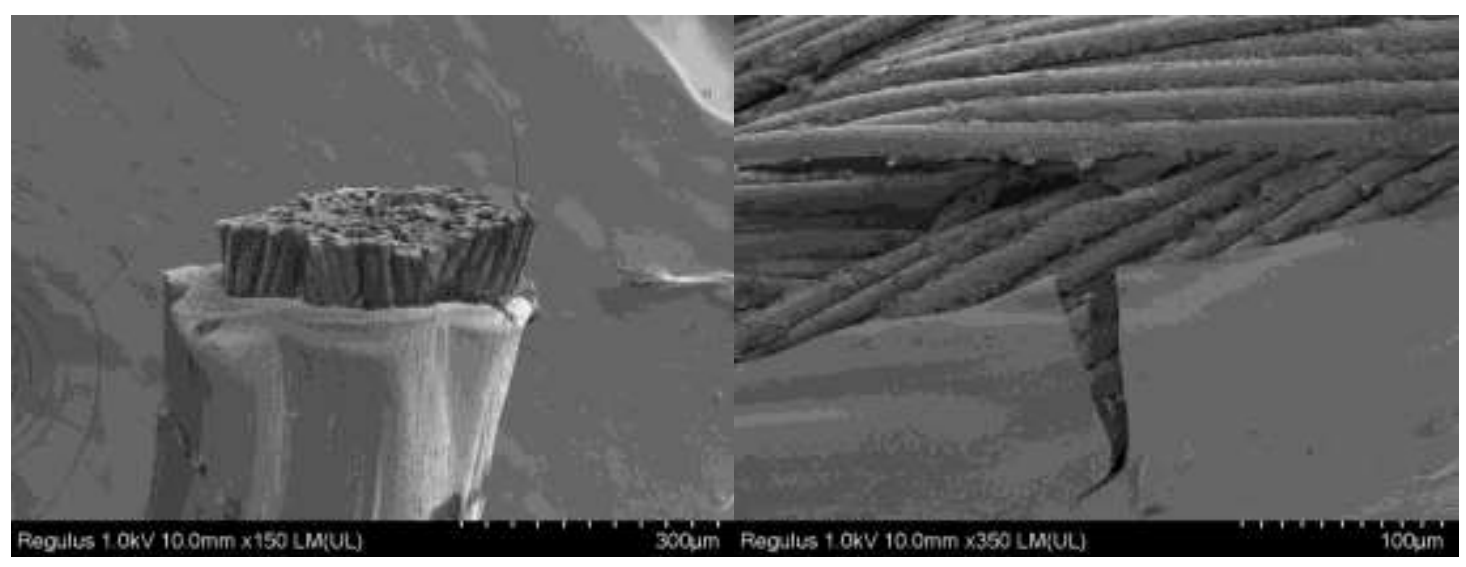

Figure 2. Structural analysis of core/shell composite (suture/hydrogel) fibers with SEM. 
The polymeric structures and properties of hydrogels were evaluated. Drug release studies were carried out in order to examine the potential biomedical applicability of these alginate-based hydrogels. Toluidine Blue was used as first model drug for release studies. According to the obtained results, it was found that the releases of toluidine blue showed changes with increasing the alginate ratio. It is seen that the toluidine blue release capacity decreases with the increase in the density of the alginate. There was not any positive effect on toluidine blue release by adding gelatin to the alginate. Only hydrogel formed with 5\% alginate $+1 \%$ gelatin mixture showed approximately $10 \%$ increase in the drug release (Table 2). Although different ratio of starch had different effects, it generally had a negative effect on toluidine blue release.

Table 2. Release profile for hydrogels (alginate and its mixtures with starch or gelatin) of toluidine blue (TB) and Penicillin/Streptomycin (Pen_Strep).

\begin{tabular}{l|c|c}
\hline \multicolumn{1}{c|}{ HYDROGEL } & TB & PEN/STREP \\
\hline 1\% Alginate & 3.00 & 2.71 \\
\hline 1\% Alginate + 1\% Gelatin & 2.10 & 1.10 \\
\hline 1\%Alginate + 1\% Starch & 3.12 & 3.01 \\
\hline 2\%Alginate + 2\% Gelatin & 2.02 & 1.98 \\
\hline 2\%Alginate + 2\% Starch & 2.61 & 2.01 \\
\hline 3\%Alginate & 2.17 & 1.22 \\
\hline 3\%Alginate + 2\% Gelatin & 1.66 & 1.44 \\
\hline 3\%Alginate + 2\% Starch & 1.53 & 1.53 \\
\hline 5\%Alginate & 1.05 & 1.02 \\
\hline 5\%Alginate + 1\% Gelatin & 1.15 & 1.01 \\
\hline
\end{tabular}

In the case of penicillin streptomycin, it is observed that the release of the drug from alginate-gelatin hydrogels is lower than the alginate hydrogel when the ratio of alginate is 1 or $2 \%$ (gelatin ratio is the same as alginate $1 \%: 1 \%$ or $2 \%: 2 \%$ ). But with increase the ratio of alginate to 3 or $5 \%$ the drug release amount increases by increasing the gelatin ratio to $2 \%$.

In different studies, swelling tests of alginate: gelatin mixtures at different doses were performed. The common opinion of the studies has been shown that the swelling levels increase with the addition of gelatin[40-42]. The reason of the converse results for lower ratio of alginate (1 and 2\%) in our study is thought to be the used $\mathrm{CaCl}_{2}$ concentration and the difference in chemical crosslinker.

There is no correlation in the toluidine blue release profile in the hydrogels obtained by adding starch. However, in penicillin streptomycin release amounts, high levels of release were observed by adding starch in compare with hydrogel composed only from alginate. This result is thought to be due to the fact that starch is a water-insoluble material, and that molecules are trapped in the voids during polymerization, creating a higher surface area.

Surgicalsutures coated with MSC loaded alginate were labeled with PKH26 (red). Fluorescent microscope imaging of these sutures shows the presence of MSCs in shell part of the fibers. Surgical sutures are coated with alginate in order to cover them with a cell-loaded hydrogel layer in the form of a core-shell. For crosslinking process of the alginate, the coated fibril (surgical suture + MSC-alginate) was kept in $\mathrm{CaCl}_{2}$ solution for about 2 minutes and then imagined under the fluorescent microscope (Figure 3). As seen in Figure 3, hAT-MSCs showed a homogeneous distribution. In core/shell composite fibers, it has been shown that the shell of alginate-based blend hydrogel polymers can encapsulate mesenchymal stem cells with high viability rate. 


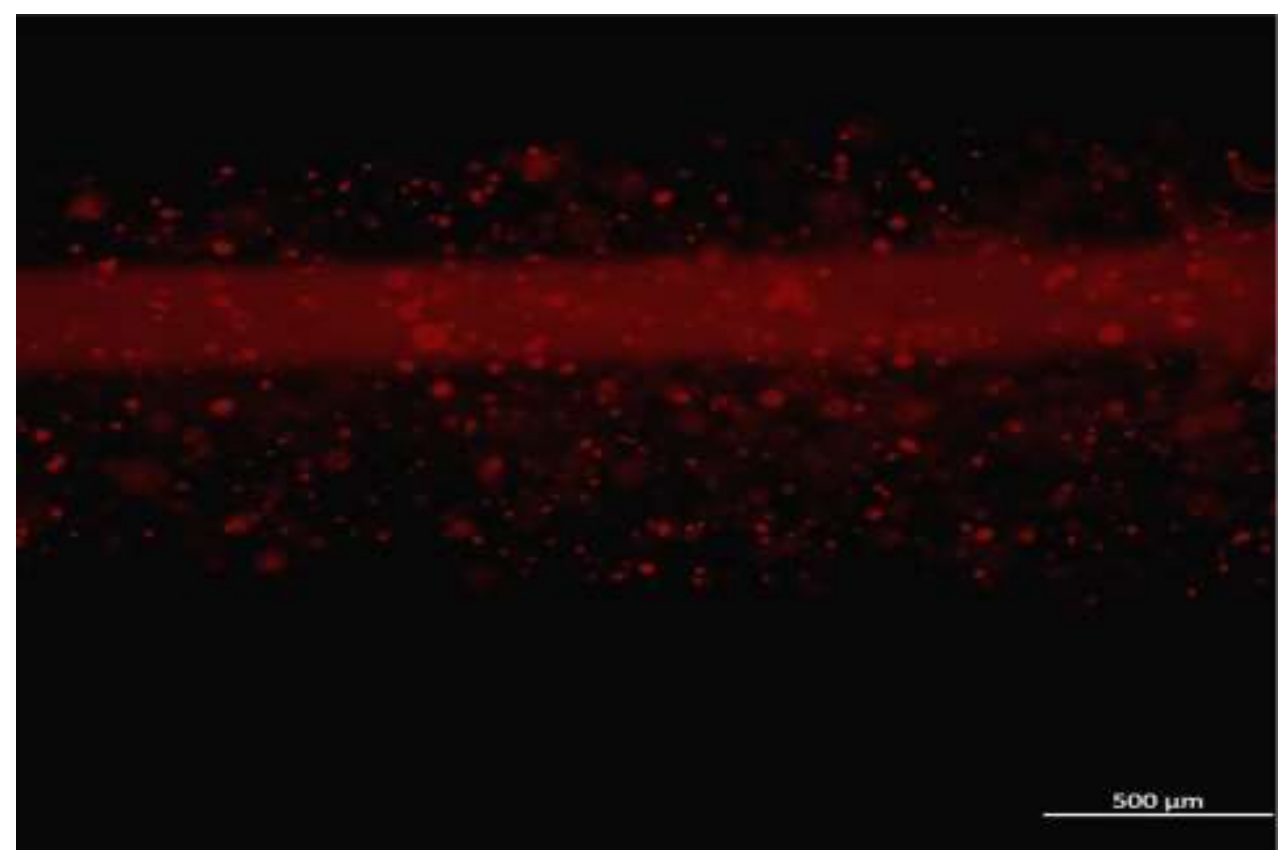

Figure 3. Fluorescent microscope image of live composite suture coated with Mesenchymal Stem Cell loaded \%3Alginate:\%2Gelatin labeled with PKH26 (red) in a core-shell structure.

The data we obtained in this study showed that not only the swelling and drug release profile of alginate and starch or gelatin added alginate hydrogels can be controlled, butalso, it has been shown that living cells can adhere to the alginate hydrogel and maintain their vitality within hydrogel. Due to these properties, alginate and starch or gelatin doped alginate hydrogels appear as low-cost polymers that can be used in biomedical applications. Nowadays, biomedical studies are mostly focused on biomaterials such as hyaluronic acid, cellulose and its derivatives, and chitosan. This studyshowed that alginate and alginate-based materials to be used directly, it should be both low cost and highly effective. It is thought that alginate and its mixtures, which have been shown to be effective both in cost and in the data of our study, can be developed further with future studies to produce medically usable living products.
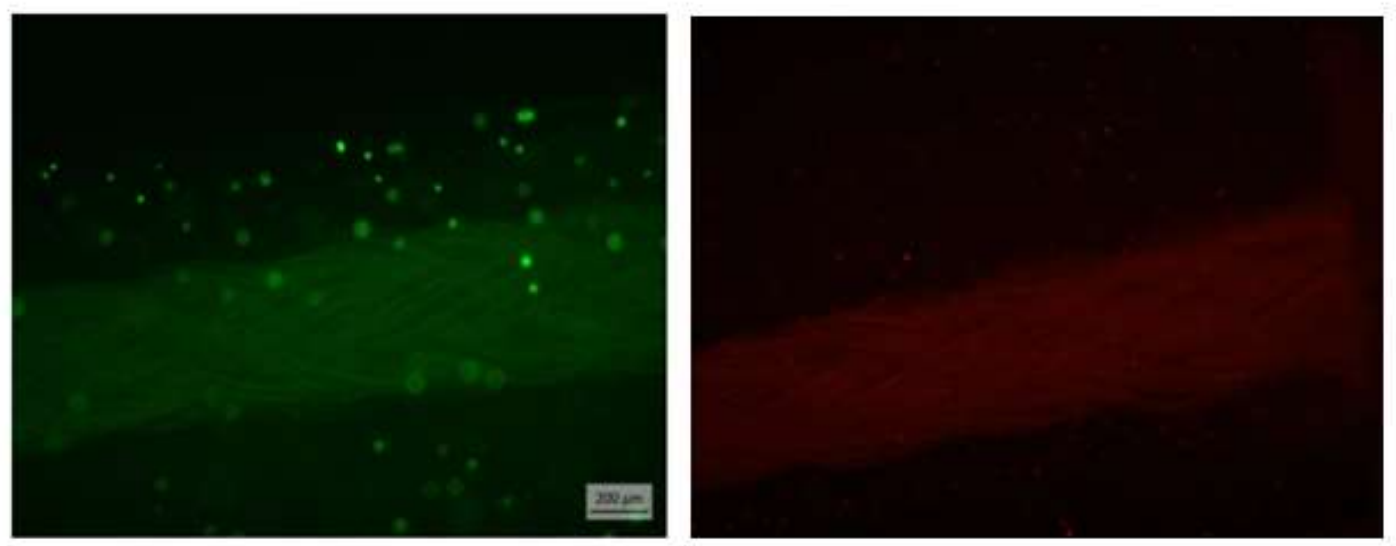

Figure 4. Biocompatibility assessment of the core-shell fiber construction process with live/dead cell viability assay. Fluorescence images of human adipose tissue-derived mesenchymal stem cells (hAT-MSCs) encapsulated within composite fibers. 3D core-shell was generated from surgical sutures coated with 
\%3Alg:\%2Gel hydrogel blend were imaged post-24 hours cell culture. Scale bars, (a) and (b) $200 \mu \mathrm{m}$. (a), Live cells are green (calcein acetoxymethyl) and (b) dead cells are red (propidium iodide).

In this study, biocompatibility of core-shell fiber preparation process with alginate: gelatin blends has been demonstrated by live dead staining. Cell viability of composite fibers was evaluated by using hAT-MSCs in shell part of composite fibers. The high cellular viability after 24 hours of culture indicates that living core-shell fiber preparation process did not cause any significant cytotoxic effect to the cells. This result indicated that encapsulated cells remained alive after 24 hours(Figure $4 \mathrm{a}, 4 \mathrm{~b}$,). In a reported study regarding composite fibers, more than $50 \%$ cells was found dead after 7 days culture from encapsulationwith hydrogel layer was composed of alginate[43]. Alginate may not be suitable for long-term culture, but it is a suitable biomaterial for encapsulating cells. Since, it can be used with cell-friendly biomaterials (i.e. gelatin, collagen, fibronectin etc.) as blend formulations, while enabling shape fidelity. Alginate and gelatin can be used as primary coating materials as composite form due to favorable rheological properties[44]. Although alginate is a biocompatible material and adjustable properties with greater mechanical stiffness and strength among the other biomaterials, it is inert material for cellularattachmentdue to the lack of essential amino acid sequences (RGD). Thus,it is not appropriate to use alginate alone as ECMin tissue engineering approaches [45].For maintaining biological activities such as cell adhesion, proliferation, migration, growth,peptide containing-hydrogels have been studied for decades [46]. Gelatin can be suggested as bi-functional coating material as biocompatible and biodegradable, since it possessRDG motifs naturally for cellularadhesion, and it can be easily degraded by cellswith particularly MMP enzymes that is released from cells[47]. However, when gelatin is exposed to heat, its lossshape fidelity. Using gelatinwith alginate at the appropriate concentration prevents this mechanical stability issue.According to a recent study by Wang et al. (2018), the viability of human adipose-derived stem cells within the alginate/gelatin mixture was evaluated, it has shown that alginate/gelatin mixture did not have any cytotoxic effect on cells and is suitable for cell proliferation[48].

In another study conducted with gelatin derivatives: alginate containing composite fibers in 2019, it was shown that the encapsulated cells remained viable for up to 21 days[4]. Cell survival and proliferation has positive correlation with gelatin concentration, whereas negative correlation with alginate concentration. On the other hand lower alginate percentage shape fidelity and stability of material during coating process with a suitable viscosity[48]. Therefore, based on these results in our study, $3 \%$ alginate: $2 \%$ gelatinratio was used for fabrication composite living fibers.

\section{CONCLUSIONS}

The results of this study showed that the swelling and drug release profiles of alginate and starch or gelatin doped hydrogels can be controlled. Also, it has been shown that cell survival can bemaintained in the alginate hydrogel with high viability. Due to these properties, alginate and doped alginate hydrogels appear as low-cost polymers that can be used in biomedical applications. Recent biomedical studies are mostly focused on biomaterials such as hyaluronic acid,chitosan, cellulose and its derivatives. This study showed that it would be advantageous to usealginate-based materials in the living composite fibers for biomedical studies, in terms of low cost and high efficacy. It is thought that alginate and its mixtures, which have been shown to be effective in our study, can be developed further with future studies to produce medically usable products.

\section{ACKNOWLEDGMENTS}

This study is supported by Turkish Scientific and Technological Council (TÜBİTAK-1002) under the grant number of 219 S647. 


\section{CONFLICT OF INTEREST}

The authors stated that there are no conflicts of interest regarding the publication of this article.

\section{REFERENCES}

[1] Martin P. Wound Healing--Aiming for Perfect Skin Regeneration. Science, 1997; 276: 75-81.

[2] Gonzalez ACDO, Costa TF, Andrade ZDA \& Medrado, ARAP. Wound Healing-A Literature Review. Anais Brasileiros de Dermatologia 2016; 91:614-620.

[3] Tatara AM, Kontoyiannis DP, Mikos AG. Drug Delivery and Tissue Engineering to Promote Wound Healing in the Immunocompromised Host: Current Challenges and Future Directions. Advanced Drug Delivery Reviews, 2018; 129: 319-329.

[4] Costa- Almeida R, Domingues RM, Fallahi A, Avci H, Yazdi IK, Akbari M, et al. Cell- Laden Composite Suture Threads for Repairing Damaged Tendons. Journal of tissue Engineering and Regenerative Medicine, 2018; 12:1039-1048.

[5] Fallahi A, Yazdi IK, Serex L, Lesha E, Faramarzi N, Tarlan F, et al. Customizable Composite Fibers for Engineering Skeletal Muscle Models. ACS Biomaterials Science \& Engineering 2019; 6:1112-1123.

[6] Tamayol A, Yazdi I, Fallahi A, Nabavinia M, Avci H, Costa-Almeida R, et al. Textile Tissue Engineering: a Path Towards Organ Weaving. in Front. Bioeng. Biotechnol. Conference Abstract: 10th World Biomaterials Congress FBIOE, 2016 doi: 10.3389/conf..

[7] Costa-Almeida R, Tamayol A, Yazdi I, Avci H, Fallahi A, Annabi N. et al. A Textile Platform using Mechanically Reinforced Hydrogel Fibres Towards Engineering Tendon Niche, European Cells and Materials, 2016; 31: 1473-2262.

[8] Adeli H, Khorasani MT and Parvazinia MJI. Wound Dressing Based on Electrospun PVA/Chitosan/Starch Nanofibrous Mats: Fabrication, Antibacterial and Cytocompatibility Evaluation and in vitro Healing Assay. International Journal of Biological Macromolecules, 2019; 122: 238-254.

[9] Baghaie S, Khorasani MT, Zarrabi A and Moshtaghian JJJ. Wound Healing Properties of PVA/Starch/Chitosan Hydrogel Membranes with Nano Zinc Oxide as antibacterial Wound Dressing Material. Polymer Edition, 2017; 28:2220-2241.

[10] Zhang M \& Zhao X. Alginate Hydrogel Dressings for Advanced Wound Management. International Journal of Biological Macromolecules, 2020; 162: 1414-1428

[11] Mayet N, Choonara YE, Kumar P, Tomar LK, Tyagi C, Toit L. C. Du, et al. A comprehensive review of advanced biopolymeric wound healing systems. Journal of Pharmaceutical Sciences 2014; 103: 2211-2230.

[12] Lee KY, Mooney DJ. Alginate: Properties and Biomedical Applications. Progress in Polymer Science, 2012;37: 106-126. 
[13] Gaspar-Pintiliescu A, Stanciuc AM and Craciunescu O. Natural Composite Dressings Based on Collagen, Gelatin and Plant Bioactive Compounds for Wound Healing: A Review. International Journal of Biological Macromolecules, 2019;138: 854-865.

[14] Hasnain MS, Nayak AK. Alginates: Versatile Polymers in Biomedical Applications and Therapeutics. CRC Press, 2019.

[15] Sudha PN. Industrial Applications Of Marine Biopolymers. CRC Press, 2017.

[16] Mir M, Ali MN, Barakullah A, Gulzar A, Arshad M, Fatima S, et al. Synthetic Polymeric Biomaterials for Wound Healing: A Review. Progress in Biomaterials, 2018; 7:1-21.

[17] Christensen BE. Alginates as Biomaterials in Tissue Engineering. Carbohydrate Chemistry: Chemical and Biological Approaches, 2011; 37:227-258.

[18] Torres FG, Commeaux S \& Troncoso OP. Starch- Based Biomaterials for Wound- Dressing Applications. Starch- Stärke, 2013; 65: 543-551.

[19] Naseri-Nosar M, Ziora ZM. Wound Dressings from Naturally-Occurring Polymers: A Review On Homopolysaccharide-Based Composites. Carbohydrate Polymers, 2018; 189:379-398.

[20] Campbell FC. Structural Composite Materials. ASM international, 2010.

[21] Malafaya PB, Silva GA \& Reis RL. Natural-Origin Polymers as Carriers and Scaffolds for Biomolecules and Cell Delivery in Tissue Engineering Applications. Advanced Drug Delivery Reviews, 2007; 59: 207-233.

[22] Pourjavadi A, Ebrahimi AA \& Barzegar, S. Preparation and Evaluation of Bioactive and Compatible Starch Based Superabsorbent for Oral Drug Delivery Systems. Journal of Drug Delivery Science and Technology, 2013; 23:511-517.

[23] Zhang LM, Yang C \& Yan L. Perspectives on: Strategies to Fabricate Starch-Based Hydrogels with Potential Biomedical Applications. Journal of Bioactive and Compatible Polymers, 2005; 20: 297-314.

[24] Afewerki S, Sheikhi A, Kannan S, Ahadian S \& Khademhosseini A. Gelatin- Polysaccharide Composite Scaffolds for 3D Cell Culture and Tissue Engineering: Towards Natural Therapeutics. Bioengineering \& Translational Medicine, 2019; 4: 96-115.

[25] Choi YS, Hong S R, Lee YM, Song KW, Park MH \& Nam YS. Study on Gelatin-Containing Artificial Skin: I. Preparation and Characteristics of Novel Gelatin-Alginate Sponge. Biomaterials, 1999; 20: 409-417.

[26] Princely S, Saleem Basha N, Nandhakumar S and Dhanaraju MJSRL. Design and Evaluation of Controlled Release Gentamycin Incorporated Gelatine Alginate Matrices for Wound Management. Scholars Research Library, 2015; 7:145-153.

[27] Abdullah MF, Nuge T, Andriyana A, Ang BC and Muhamad, F. Core-Shell Fibers: Design, Roles, and Controllable Release Strategies in Tissue Engineering and Drug Delivery. Polymers, 2019; 11:2008 
[28] Avci H, Ghorbanpoor H and Nurbas M. Preparation of Origanum Minutiflorum Oil-Loaded Core-Shell Structured Chitosan Nanofibers with Tunable Properties. Polymer Bulletin, 2018; 75:4129-4144.

[29] Karaöz E, Demircan PÇ, Erman G, Güngörürler E and Sarıboyacı AE. Comparative Analyses of Immunosuppressive Characteristics of Bone-Marrow, Wharton's Jelly, and Adipose TissueDerived Human Mesenchymal Stem Cells. Turkish Journal of Hematology, 2017; 34:213.

[30] Özdemir AT, Özdemir RBÖ, Kırmaz C, Sarıboyacı AE, Halbutoğlları ZSÜ, Özel C, et al. The Paracrine Immunomodulatory Interactions Between The Human Dental Pulp Derived Mesenchymal Stem Cells and CD4 T Cell Subsets. Cellular Immunology, 2016; 310:108-115.

[31] Özdemir RBÖ, Özdemir AT, Sarıboyacı AE, Uysal O, Tuğlu Mİ and Kırmaz C. The Investigation of Immunomodulatory Effects of Adipose Tissue Mesenchymal Stem Cell Educated Macrophages on The CD4 T Cells. Immunobiology, 2019; 224:585-594.

[32] Zhuang Y, Yu F, Chen H, Zheng J, Ma J and Chen J. Alginate/Graphene Double-Network Nanocomposite Hydrogel Beads with Low-Swelling, Enhanced Mechanical Properties and Enhanced Adsorption Capacity. Journal of Materials Chemistry A, 2016; 4:10885-10892.

[33] Matyash M, Despang F, Ikonomidou C and Gelinsky M. Swelling and Mechanical Properties of Alginate hydrogels with Respect to Promotion of Neural Growth. Tissue Engineering Part C: Methods 2014; 20:401-411.

[34] Bajpai S and Kirar N. Swelling and Drug Release Behavior of Calcium Alginate/Poly (Sodium Acrylate) Hydrogel Beads. Designed Monomers and Polymers, 2016; 19:89-98.

[35] Roy A, Bajpai J and Bajpai A. Dynamics of Controlled Release of Chlorpyrifos from Swelling and Eroding Biopolymeric Microspheres of Calcium Alginate and Starch. Carbohydrate Polymers, 2009; 76:222-231.

[36] Yao R, Zhang R, Luan J and Lin F. Alginate and Alginate/Gelatin Microspheres for Human Adipose-Derived Stem Cell Encapsulation and Differentiation. Biofabrication, 2012; 4:025007.

[37] Pulat M. The Preparation of Gelatin Coated Sodium Alginate Hydrogels. The Eurasia Proceedings of Science Technology Engineering and Mathematics, 2018; 4:149-155

[38] El-Sherbiny I, Lins R, Abdel-Bary E and Harding D. Preparation, Characterization, Swelling and in vitro Drug Release Behaviour of Poly [N-acryloylglycine-Chitosan] Interpolymeric $\mathrm{pH}$ and Thermally-Responsive Hydrogels. European Polymer Journal, 2005; 41:2584-2591.

[39] Wang Q, Hu X, Du Y and Kennedy JF. Alginate/Starch Blend Fibers and Their Properties for Drug Controlled Release. Carbohydrate Polymers, 2010; 82:842-847.

[40] Dong Z,Wang Q and Du Y. Alginate/Gelatin Blend Films and Their Properties for Drug Controlled Release. Journal of Membrane Science, 2006; 280:37-44.

[41] Fan L, Du Y, Huang R, Wang Q, Wang X and Zhang L. Preparation and Characterization of Alginate/Gelatin Blend Fibers. Journal of Applied Polymer Science, 2005; 96:1625-1629. 
[42] Rosellini E, Cristallini C, Barbani N, Vozzi G and Giusti P. Preparation and Characterization of Alginate/Gelatin Blend Films for Cardiac Tissue Engineering. Journal of Biomedical Materials Research Part A: An Official Journal of The Society for Biomaterials, The Japanese Society for Biomaterials and The Australian Society for Biomaterials and the Korean Society for Biomaterials, 2009; 91:447-453.

[43] Akbari M, Tamayol A, Laforte V, Annabi N, Najafabadi AH, Khademhosseini A. et al. Composite Living Fibers for Creating Tissue Constructs using Textile Techniques. Advanced Functional Materials, 2014; 24:4060-4067

[44] He Y, Yang F, Zhao H, Gao Q, Xia B and Fu J. Research on The Printability of Hydrogels in 3D bioprinting. Scientific Reports, 2016; 6:1-13.

[45] Axpe E and Oyen ML. Applications of Alginate-Based Bioinks in 3D Bioprinting. International Journal of Molecular Sciences, 2016; 17:1976

[46] Wells RG. The Role of Matrix Stiffness in Regulating Cell Behavior. Hepatology, 2008; 47:1394-1400.

[47] Kalia S. Polymeric Hydrogels As Smart Biomaterials. Springer, 2016; Berlin, Germany.

[48] Wang XF, Lu PJ, Song Y, Sun YC, Wang YG and Wang Y. Nano Hydroxyapatite Particles Promote Osteogenesis in A Three-Dimensional Bio-Printing Construct Consisting of Alginate/Gelatin/hASCs. RSC Advances, 2016; 6:6832-6842. 